\title{
Effects of Rotation on Stochasticity of Gravitational Waves in Nonlinear Phase of Core-Collapse Supernovae
}

\author{
Kei Kotake ${ }^{1}$, Wakana Iwakami Nakano², and Naofumi Ohnishi ${ }^{2}$ \\ ${ }^{1}$ Division of Theoretical Astronomy, National Astronomical Observatory of Japan, 2-21-1, \\ Osawa, Mitaka, Tokyo, 181-8588, Japan \\ kkotake@th.nao.ac.jp \\ ${ }^{2}$ Department of Aerospace Engineering, Tohoku University, 6-6-01 Aramaki-Aza-Aoba, \\ Aoba-ku, Sendai, 980-8579, Japan
}

\begin{abstract}
By performing three-dimensional (3D) simulations that demonstrate the neutrino-driven core-collapse supernovae aided by the standing accretion shock instability (SASI), we study how the spiral modes of the SASI can have impacts on the properties of the gravitational-wave (GW) emission. To see the effects of rotation in the non-linear postbounce phase, we give a uniform rotation on the flow advecting from the outer boundary of the iron core, whose specific angular momentum is assumed to agree with recent stellar evolution models. We compute fifteen 3D models in which the initial angular momentum as well as the input neutrino luminosities from the protoneutron star are changed in a systematic manner. By performing a ray-tracing analysis, we accurately estimate the GW amplitudes generated by anisotropic neutrino emission. Our results show that the gravitational waveforms from neutrinos in models that include rotation exhibit a common feature otherwise they vary much more stochastically in the absence of rotation. The breaking of the stochasticity stems from the excess of the neutrino emission parallel to the spin axis. This is because the compression of matter is more enhanced in the vicinity of the equatorial plane due to the growth of the spiral SASI modes, leading to the formation of the spiral flows circulating around the spin axis with higher temperatures. We point out that a recently proposed future space interferometers like Fabry-Perot type DECIGO would permit the detection of these signals for a Galactic supernova.
\end{abstract}

Subject headings: supernovae: collapse — gravitational waves — neutrinos hydrodynamics 


\section{Introduction}

The successful detection of neutrinos from SN1987A paved the way for Neutrino Astronomy (Hirata et al. 1987), an alternative to conventional astronomy by electromagnetic waves. Core-collapse supernovae are now expected to be opening yet another astronomy, Gravitational-Wave Astronomy. Currently long-baseline laser interferometers such as LIGO (Abbott et al. 2005), VIRGO ${ }^{1}$, GEO600 $^{2}$, and TAMA300 (Ando \& the TAMA Collaboration 2005) are operational (see, e.g., Hough et al. (2005) for a recent review). For these detectors, core-collapse supernovae have been proposed as one of the most plausible sources of gravitational waves (GWs) (see, for example, Kotake et al. (2006); Ott (2009) for recent reviews).

Although the mechanism of explosion is not completely understood yet, current multidimensional simulations based on refined numerical models show several promising scenarios. Among the candidates is the neutrino heating mechanism aided by convection and standing accretion shock instability (SASI) (e.g., Marek \& Janka (2009); Bruenn et al. (2010); Suwa et al. (2009)), the acoustic mechanism (Burrows et al. 2006), or the magnetohydrodynamic (MHD) mechanism (e.g., Takiwaki et al. (2004, 2009); Obergaulinger et al. (2006); Burrows et al. (2007), and Kotake et al. (2006) for collective references therein). For the former two to be the case, the explosion geometry is expected to be unipolar and bipolar, and for the MHD mechanism to be bipolar. Since the GW signatures imprint a live information of the asphericity at the moment of explosion, they could provide us invaluable clues to understand the supernova mechanism.

Traditionally, most of the theoretical predictions of GWs have focused on the bounce signals (e.g., Mönchmever et al. (1991); Zwerger \& Müller (1997); Kotake et al. (2003, 2004); Shibata \& Sekiguchi (2004); Ott et al. (2004,, 2007a,.c); Dimmelmeier et al. (2002, 2007, 2008); Scheidegger et al. (2010)) and references therein). However recent stellar evolution calculations suggest that rapid rotation assumed in most of the previous studies is not canonical for progenitors with neutron star formations (Heger et al. 2005; Ott et al. 2006b). Besides the rapid rotation of the core, convective matter motions and anisotropic neutrino emission in the much later postbounce phase are expected to be the primary GW sources with comparable amplitudes to the bounce signals. Thus far, various physical ingredients for producing asphericities and the resulting GWs in the postbounce phase have been studied, such as the roles of pre-collapse density inhomogeneities (Burrows \& Haves 1996; Müler \& Janka

\footnotetext{
${ }^{1}$ http://www.ego-gw.it/

${ }^{2}$ http://geo600.aei.mpg.de/
} 
1997; Fryer 2004a, b), moderate rotation of the iron core (Müller et al. 2004), nonaxisymmetric rotational instabilities (Rampp et al. 1998; Ott et al. 2007b), g-modes (Ott et al. 2006a) and r-modes pulsations (Andersson et al. 2010) of protoneutron stars (PNSs), and SASI (Kotake et al. (2007, 2009a,b); Marek et al. (2009); Murphy et al. (2009)).

Here SASI, becoming very popular in current supernova researches, is a uni- and bipolar sloshing of the stalled supernova shock with pulsational strong expansion and contraction (e.g., Blondin et al. (2003); Scheck et al. (2004); Ohnishi et al. (2006); Foglizzo et al. (2007); Iwakami et al. (2008)). Based on two-dimensional (2D) simulations that parametrize the neutrino heating and cooling by a light-bulb scheme to obtain explosions (which we shortly call as parametric SASI simulations), we pointed out that the GW amplitudes from anisotropic neutrino emission increase almost monotonically with time, and that such signals may be visible to next-generation detectors for a Galactic source (Kotake et al. 2007, 2009a). By performing such a parametric simulation but without the excision inside the PNS, Murphy et al. (2009) showed that the GW signals from matter motions can be a good indicator to get the information of the explosion geometry. These features qualitatively agree with the ones obtained by Yakunin et al. (2010) who reported exploding 2D simulations in which a multi-group flux limited diffusion transport is solved with the hydrodynamics. Marek et al. (2009) analyzed the GW emission based on their long-term 2D (ray-by-ray) Boltzmann simulations, which seem very close to produce explosions (Marek \& Janka 2009). They also confirmed that the GWs from neutrinos with continuously growing amplitudes (but with the different sign of the amplitudes in Kotake et al. (2007, 2009a); Yakunin et al. $(2010)$ ), are dominant over the ones from matter motions. They proposed that the thirdgeneration class detectors such as the Einstein Telescope are required for detecting the GW signals with a good signal-to-noise ratio.

Thanks to a growing computational power, three-dimensional (3D) simulations, though mostly limited to the parametric simulations at present, are now becoming practicable. In 3D, the modes of SASI are divided into sloshing modes and spiral modes (e.g., Iwakami et al. (2008)). Asymmetric $m=0$ modes so far studied in 2D models and axisymmetric $m \neq 0$ modes are classified into the sloshing modes, where $m$ stands for the azimuthal index of the spherical harmonics $Y_{l}^{m}$. In the latter situation, the $\pm m$ modes degenerate so that the $+m$ modes has the same amplitudes as the $-m$ modes. If random perturbations or uniformly rotating flow are imposed on these axisymmetric flows in the postbounce phase, the degeneracy is broken and the rotational modes emerge (Blondin \& Mezzacappa 2007; Iwakami et al. 2009). In this situation, the $+m$ modes has the different amplitudes from $-m$ modes. Such rotating non-axisymmetric $m \neq 0$ modes are called as spiral modes. These nonaxisymmetric modes are expected to bring about a breakthrough in our supernova theory, because they can help to produce explosions more easily compared to $2 \mathrm{D}$ due to its extra 
degree of freedom (Nordhaus et al. 2010), and also because they may have a potential to generate pulsar spins (Blondin \& Mezzacappa (2007), see also Yamasaki \& Foglizzo (2008); Wongwathanarat et al. (2010)).

In a series of our previous papers, we have studied the effects of the sloshing SASI modes on the GW signatures based on the 2D parametric SASI simulations (Kotake et al. 2007, 2009a). In the latter study, we proposed a ray-tracing method to accurately estimate GWs generated by anisotropic neutrino emission. Then moving on to the 3D non-rotating parametric simulations, we pointed out that the gravitational waveforms in 3D vary much more stochastically than for the corresponding 2D models because the explosion anisotropies depend sensitively on the growth of the SASI which develops chaotically in all directions (Kotake et al. 2009b). As a sequel of these studies, we hope to study the role of the spiral SASI modes on the GW emission in this work. We include the effects of rotation as in Iwakami et al. (2009). That is, when the SASI gradually transits from the linear to nonlinear phase, we give a uniform rotation to the flow advecting from the outer boundary of the iron core, whose specific angular momentum is assumed to agree with recent stellar evolution models (Heger et al. 2005). After the initial rotational flows advect to the PNS surface, rotation begins to have influence over the GW signals due to the non-axisymmetric flow motions and anisotropic neutrino emission outside the PNS. Such an approach (excision inside the PNS), could be justified by some striking evidences that support the slow rotation of iron cores (Heger et al. 2005), and also that the postbounce density structure of the PNS for such a progenitor (model m15b6 in Heger et al. (2005)) has a very similar structure to the ones in the non-rotating models (Ott et al. 2006b). To see clearly the effects of rotation, we compute fifteen $3 \mathrm{D}$ models in which the initial angular momentum as well as the input neutrino luminosities from the PNS are changed in a systematic manner. To estimate the GWs from anisotropic neutrino radiation, we perform the ray-tracing analysis. With these computations, we hope to clarify how rotation that gives a special direction to the system (i.e., the spin axis), could affect the stochastic GW features obtained in our previous study (e.g., Kotake et al. (2009b)).

The plan of this paper is as follows. In section 2, we shortly summarize the information how to construct our 3D models and also how to compute the gravitational waveforms. The main results are shown in Section 3. We summarize our results and discuss their implications in Section 4 . 


\section{Numerical Methods and Models}

\subsection{Extraction of Gravitational Waveforms}

The numerical methods to extract the gravitational waveforms have been already described in Kotake et al. (2009a). We extract the GW amplitudes from mass motions using the standard quadrupole formula (e.g., Zhuge et al. (1994); Rampp et al. (1998)), and their

spectra by Flanagan \& Hughes (1998) with the FFT techniques. For the GWs generated by anisotropic neutrino emission (Epstein 1978; Müler \& Janka 1997), the two modes of the GWs can be derived as follows,

$$
\begin{aligned}
h_{+}= & C \int_{0}^{t} \int_{4 \pi} d \Omega^{\prime}\left(1+s\left(\theta^{\prime}\right) c\left(\phi^{\prime}\right) s(\xi)+c\left(\theta^{\prime}\right) c(\xi)\right) \times \\
& \frac{\left(s\left(\theta^{\prime}\right) c\left(\phi^{\prime}\right) c(\xi)-c\left(\theta^{\prime}\right) s(\xi)\right)^{2}-s^{2}\left(\theta^{\prime}\right) s^{2}\left(\phi^{\prime}\right)}{\left[s\left(\theta^{\prime}\right) c\left(\phi^{\prime}\right) c(\xi)-c\left(\theta^{\prime}\right) s(\xi)\right]^{2}+s^{2}\left(\theta^{\prime}\right) s^{2}\left(\phi^{\prime}\right)} \frac{d l_{\nu}\left(\Omega^{\prime}, t^{\prime}\right)}{d \Omega^{\prime}},
\end{aligned}
$$

and

$$
\begin{aligned}
h_{\times}= & 2 C \int_{0}^{t} \int_{4 \pi} d \Omega^{\prime}\left(1+s\left(\theta^{\prime}\right) c\left(\phi^{\prime}\right) s(\xi)+c\left(\theta^{\prime}\right) c(\xi)\right) \times \\
& \frac{s\left(\theta^{\prime}\right) s\left(\phi^{\prime}\right)\left(s\left(\theta^{\prime}\right) c\left(\phi^{\prime}\right) c(\xi)-c\left(\theta^{\prime}\right) s(\xi)\right)}{\left[s\left(\theta^{\prime}\right) c\left(\phi^{\prime}\right) c(\xi)-c\left(\theta^{\prime}\right) s(\xi)\right]^{2}+s^{2}\left(\theta^{\prime}\right) s^{2}\left(\phi^{\prime}\right)} \frac{d l_{\nu}\left(\Omega^{\prime}, t^{\prime}\right)}{d \Omega^{\prime}},
\end{aligned}
$$

where $s(A) \equiv \sin (A), c(B) \equiv \cos B, C \equiv 2 G /\left(c^{4} R\right)$ with $G, c$ and $R$, being the gravitational constant, the speed of light, the distance of the source to the observer respectively, $d l_{\nu} / d \Omega$ represents the direction-dependent neutrino luminosity emitted per unit of solid angle into direction of $\Omega$, and $\xi$ is the viewing angle (e.g., Kotake et al. (2009a)). For simplicity, we consider here two cases, in which the observer is assumed to be situated along 'polar' $(\xi=0)$ or 'equatorial' $(\xi=\pi / 2)$ direction. It is important to note that in the case of $2 \mathrm{D}$ axisymmetric case, the only non-vanishing component is the plus mode for the equatorial observer,

$$
h_{+}^{\mathrm{e}}=2 C \int_{0}^{t} d t^{\prime} \int_{0}^{\pi} d \theta^{\prime} \Phi\left(\theta^{\prime}\right) \frac{d l_{\nu}\left(\theta^{\prime}, t^{\prime}\right)}{d \Omega^{\prime}}
$$

where the function of $\Phi\left(\theta^{\prime}\right)$ has positive values in the north polar cap for $0 \leq \theta^{\prime} \leq 60^{\circ}$ and in the south polar cap for $120^{\circ} \leq \theta^{\prime} \leq 180^{\circ}$, but becomes negative values between $60^{\circ}<\theta^{\prime}<120^{\circ}$ (see Figure 1 of Kotake et al. (2007)).

To determine $d l_{\nu} / d \Omega$ in equations (1, 2), we perform a ray-tracing calculation which we shortly summarize in the following (see Kotake et al. (2009a) for more detail). In the ray-tracing approach, we consider transfer along the ray specified by a constant impact parameter $p$. The coordinate along $p$ is called $s$, satisfying

$$
r=\left(p^{2}+s^{2}\right)^{1 / 2}
$$


where $r$ is the radial coordinate. In order to get the numerical convergence of $d l_{\nu}(\Omega) / d \Omega$, we need to set 45,000 rays for each direction, which consists of $500 \times 90$ rays, where the former is for the impact parameters covering from the inner- $\left(p_{\text {in }}=50 \mathrm{~km}\right)$ to the outerboundary $\left(p_{\text {out }}=2000 \mathrm{~km}\right.$ ) of the computational domain and the latter is for covering the circumference (e.g., $2 \pi$ ) of the concentric circles on the plane perpendicular to the rays.

The transfer equation of the neutrino occupation probability $f_{\nu}\left(\epsilon_{\nu}, p, s\right)$ for a given neutrino energy $\epsilon_{\nu}$ along each ray is given by,

$$
\frac{d f_{\nu}\left(\epsilon_{\nu}, p, s\right)}{d s}=j\left(\epsilon_{\nu}, p, s\right)\left(1-f_{\nu}\left(\epsilon_{\nu}, p, s\right)\right)-\frac{f_{\nu}\left(\epsilon_{\nu}, p, s\right)}{\lambda},
$$

where $j$ and $\lambda$ is the emissivity and absorptivity via neutrino absorptions and emission by free nucleons $\left(\nu_{\mathrm{e}}+\mathrm{n} \rightleftarrows \mathrm{e}^{-}+\mathrm{p}\right)($ Bruenn (1985); Ohnishi et al. (2006)), which are dominant processes outside the PNSs. The optical depth for those reactions are estimated by $\tau_{\nu}=$ $\int_{r}^{\infty} 1 / \lambda$. For the sake of simplicity, the neutrino scattering and the velocity-dependent terms in the transport equation are neglected. Along each ray, $f_{\nu}$ is transferred by the line integral. When the line integral starts from the surface on the PNS, we set the initial value of

$$
f\left(\epsilon_{\nu}\right)=\frac{1}{1+\exp \left(\epsilon_{\nu} / k_{\mathrm{B}} T_{\nu}\right)} \cdot \frac{1}{4 \pi},
$$

assuming that the neutrino distribution function at the surface is approximated by the FermiDirac distribution with a vanishing chemical potential. Here the neutrino temperature is set to be constant near $T_{\nu_{\mathrm{e}}}=4 \mathrm{MeV}$, whose values change slightly depending on the input neutrino luminosity. Note that these values are constant in time for each model. This is necessary to realize the steady unperturbed initial states (e.g., Ohnishi et al. (2006)). For the rays that do not hit the PNS, we start the line integral from the outer most boundary antipodal to the line of sight, where $f_{\nu}$ is essentially zero.

By a post process, we perform the line integral up to the outer-most boundary for each hydro-timestep. With $f\left(\epsilon_{\nu}, p, s_{\text {out }}\right)$, which is obtained by the line integral up to the outer-most boundary, the neutrino energy fluxes along a specified direction of $\Omega$ can be estimated,

$$
\frac{d l_{\nu}(\Omega, p)}{d \Omega d S}=\int f\left(\epsilon_{\nu}, p, s_{\text {out }}\right) \cdot\left(c \epsilon_{\nu}\right) \cdot \frac{\epsilon_{\nu}^{2} d \epsilon_{\nu}}{(2 \pi \hbar c)^{3}} .
$$

By summing up the energy fluxes with the weight of the area in the plane perpendicular to the rays, we can find $d l_{\nu} / d \Omega$ along a specified direction $\Omega$,

$$
\frac{d l_{\nu}(\boldsymbol{\Omega})}{d \Omega}=\int \frac{d l_{\nu}(\boldsymbol{\Omega}, p)}{d \Omega d S} d S=\int_{p_{\text {in }}}^{p_{\text {out }}} d p 2 \pi p \frac{d l_{\nu}(\boldsymbol{\Omega}, p)}{d \Omega d S}
$$

Repeating the above procedures, $d l_{\nu}(\Omega) / d \Omega$ can be estimated for all the directions, by which we can find the amplitudes of the neutrino GWs through equations (11,2). In the following 
computations, we assume that the distance to the GW source is comparable to our galactic center $(R=10 \mathrm{kpc})$ unless stated otherwise.

\subsection{Construction of 3D Models with Rotation}

The employed numerical methods and model concepts are essentially the same as those in our previous paper (Iwakami et al. 2009). Using the ZEUS-MP code (Haves et al. 2006) as a hydro-solver, we solve the dynamics of the standing accretion shock flows of matter attracted by the protoneutron star and irradiated by neutrinos emitted from the PNS. We employ the so-called light-bulb approximation (see, e.g., Janka \& Müller (1996); Ohnishi et al. (2006)) and adjust the neutrino luminosities from the PNSs to trigger explosions. This method makes it possible for us to study the properties of GWs in the postbounce phase, namely from the shock-stall, through the growth of the SASI, to 3D explosions (see also Nordhaus et al. (2010); Wongwathanarat et al. (2010)), and is found to work well in 2D, capturing the essential features obtained by more realistic simulations (see references in Scheck et al. $(2004,2006))$.

The computational grid is comprised of 300 logarithmically spaced, radial zones to cover from the absorbing inner boundary of $\sim 50 \mathrm{~km}$ to the outer boundary of $2000 \mathrm{~km}$, and 30 polar $(\theta)$ and 60 azimuthal $(\phi)$ uniform mesh points, which are used to cover the whole solid angle (see for the resolution tests in Iwakami et al. (2008)). The initial conditions are provided in the same manner of Ohnishi et al. (2006), which describes the spherically symmetric steady accretion flow through a standing shock wave (Yamasaki \& Foglizzo 2008). In constructing the initial conditions, we assume a fixed density $\rho_{\text {in }}=10^{11} \mathrm{~g} \mathrm{~cm}^{-3}$ at the inner boundary. And the initial mass accretion rates and the initial mass of the central object are set to be $\dot{M}=1 M_{\odot} \mathrm{s}^{-1}$ and $M_{\text {in }}=1.4 M_{\odot}$, respectively. To include rotation, we impose a rigid rotation on the outer boundary of the computational domain as follows,

$$
v_{\phi}^{2 D}(r, \theta)=\beta_{\phi} v_{r}^{1 D}(r) \sin \theta,
$$

where $v_{\phi}^{2 D}$ denotes the unperturbed $\phi$ component of velocity, $v_{r}^{1 D}(r)=-\dot{M} / 4 \pi r^{2} \rho(r)$ is the unperturbed radial velocity, and $\beta_{\phi}$ denotes the rotation parameter (see Iwakami et al. (2009) for more detail). We examine the flow characteristics in the following three ways $\beta_{\phi}=0,0.01$, and 0.015 which corresponds to the specific angular momentum $L \sim(4-6) \times 10^{15} \mathrm{~cm}^{2} \mathrm{~s}^{-1}$ on the equatorial plane. This choice is close to the recent rotating presupernova models that include the effects of magnetic breaking during stellar evolution (Heger et al. 2005). It is computationally prohibitive at present to follow a long-term postbounce evolution in 3D simulations that implement the spherical coordinates due to its severe Courant-Friedlichs-Lax 
condition around the pole. As a prelude to the full 3D simulations starting from gravitational collapse to explosions without excision inside the PNS, we choose to take into account the effect of rotation by the exploratory method as mentioned above. To induce non-spherical instability we add random velocity perturbations to be less than $1 \%$ of the unperturbed radial velocity. By changing also the neutrino temperature at the PNS surface (e.g., equation (6)), we compute fifteen 3D models whose input neutrino luminosities vary in the range of $L_{\nu_{e}}=6.0-6.8 \times 10^{52} \mathrm{erg} \mathrm{s}^{-1}$ (see Table 1).

In Table1, $\Delta t$ represents the simulation time when the average shock radius that continuously increases with the growth of SASI, reaches the outer boundary of the computational domain with a typical explosion energy of $\sim 10^{51} \mathrm{erg}$. On the other hand, for models denoted by "-", we terminated the simulation at about $1000 \mathrm{~ms}$, not seeing the increase of the shock radius (represented by "No" in the table). It can be seen that models with higher neutrino luminosities such as models of series D and E produce explosions without rotation (models D0 and E0), while models with the intermediate luminosities such as models B2, C1 and C2 produce explosions only when they possess rotation (compare models B0 and C0). This is mainly because the centrifugal force induced by rotation makes not only the shock radius but also the gain region larger, which works to assist the onset of explosion. Regardless of rotation, no explosions are obtained in the lowest luminosity models (model of series A) for the rotation parameters investigated here.

To analyze clearly the role of rotation on the GW emission, models of series A that do not produce explosions, are most favorable. As will be explained more in detail later, this is because in the higher luminosity models, the violent fluid motions as well as vigorous convective overturns, which works to smear out the effects of rotation, make the analysis more complicated. For example, $\Delta t$ becomes shorter with larger angular momentum for the intermediate luminosity (models of series B and C in Table 1). However this trend is no more true for models of series D (e.g., model D2). This non-monotonic effect can be understood as a consequence of stochasticity in a regime of high neutrino luminosity.

$\alpha_{\text {sk }}$ in Table 1 represents the angle between spin and kick directions that the central PNS may receive at the final simulation time, which we estimate according to Wongwathanarat et al. (2010). The obtained spin-kick angle ranges from 25 (model B2) in which the spin direction is marginally aligned with the kick direction, $88^{\circ}$ (model E0) in which the two directions are closely perpendicular, to $170^{\circ}$ (model C1) in which the two directions are closely opposite. When a rapid rotation is imposed in our models (e.g., for our models of series 2), the final spin direction is closely aligned with the direction of the induced rotation (namely, perpendicular to the equatorial plane), while even in this case, stochasticity of the kick direction seems rather unaffected. To clarify the correlation clearly, a more elaborate study 
is needed in which the initial neutrino luminosity, perturbations, and rotation, are varied much more systematically, which we plan to study as a sequel of this study (Iwakami et al. in preparation).

As already mentioned, we can adjust the epoch by hand when the rotational flow advects to the PNS surface (see Figure 1 in Iwakami et al. (2009)). The deformation of the standing shock becomes remarkable typically at about $t=100 \mathrm{~ms}$, marking the epoch when the SASI transits from the linear to non-linear regime. For the non-exploding models of A1 and A2, we choose to inject the rotational flow so that it advects to the PNS surface at around $t=400 \mathrm{~ms}$ when the SASI has been long developed into the non-linear phase. For the other luminosity models, we take the time at around $t=100 \mathrm{~ms}$ (therefore soon after the SASI enters the non-linear phase). This is because some models with higher neutrino luminosities show a trend of explosions before the rotational flows advect to the central regions if we delay the epoch of the injection and also because we want to fix the epoch common to these models. 
Table 1. Model Summary

\begin{tabular}{|c|c|c|c|c|c|c|c|c|c|c|}
\hline Model & $\begin{array}{c}L_{\nu_{e}} \\
\left(10^{52} \mathrm{erg} / \mathrm{s}\right)\end{array}$ & $\beta_{\phi}$ & $\begin{array}{c}\Delta t \\
(\mathrm{~ms})\end{array}$ & Explosion? & $\begin{array}{l}\left|h_{\max }^{\mathrm{pol}}\right| \\
\left(10^{-22}\right)\end{array}$ & $\begin{array}{l}\left|h_{\max }^{\mathrm{equ}}\right| \\
\left(10^{-22}\right)\end{array}$ & $\begin{array}{r}\left|h_{\nu, \max }^{\mathrm{pol}}\right| \\
\left(10^{-22}\right)\end{array}$ & $\begin{array}{r}\left|h_{\nu, \max }^{\text {equ }}\right| \\
\left(10^{-22}\right)\end{array}$ & $\begin{array}{c}E_{\mathrm{GW}} \\
\left(10^{-11} M_{\odot} c^{2}\right)\end{array}$ & $\begin{array}{c}\alpha_{\text {sk }} \\
\left(^{\circ}\right)\end{array}$ \\
\hline A0 & 6.0 & 0 & - & No & $0.98(+)$ & $1.59(\times)$ & $0.72(+)$ & $1.13(\times)$ & 2.54 & 100 \\
\hline $\mathrm{A} 1$ & 6.0 & 0.01 & - & No & $1.98(+)$ & $7.62(+)$ & $1.18(+)$ & $6.74(+)$ & 6.76 & 104 \\
\hline $\mathrm{A} 2$ & 6.0 & 0.015 & - & No & $2.58(+)$ & $16.7(+)$ & $0.91(\times)$ & $15.1(+)$ & 13.4 & 63 \\
\hline B0 & 6.2 & 0 & - & No & $2.93(+)$ & $3.19(+)$ & $2.45(+)$ & $2.38(+)$ & 5.65 & 68 \\
\hline B1 & 6.2 & 0.01 & - & No & $2.88(\times)$ & $11.7(+)$ & $1.37(\times)$ & $10.3(+)$ & 15.1 & 55 \\
\hline $\mathrm{B} 2$ & 6.2 & 0.015 & 721 & Yes & $5.03(\times)$ & $11.2(+)$ & $2.86(\times)$ & $5.90(+)$ & 16.1 & 25 \\
\hline $\mathrm{C} 0$ & 6.4 & 0 & - & No & $4.11(+)$ & $5.00(+)$ & $3.46(+)$ & $4.28(+)$ & 10.7 & 41 \\
\hline $\mathrm{C} 1$ & 6.4 & 0.01 & 650 & Yes & $4.62(+)$ & $6.46(+)$ & $2.05(x)$ & $4.97(+)$ & 12.1 & 170 \\
\hline $\mathrm{C} 2$ & 6.4 & 0.015 & 515 & Yes & $3.43(+)$ & $9.38(+)$ & $1.11(\times)$ & $6.09(+)$ & 8.96 & 141 \\
\hline D0 & 6.6 & 0 & 550 & Yes & $4.31(+)$ & $2.93(+)$ & $2.25(+)$ & $2.14(\times)$ & 8.18 & 113 \\
\hline D1 & 6.6 & 0.01 & 460 & Yes & $4.73(\times)$ & $4.70(\times)$ & $3.72(\times)$ & $3.91(\times)$ & 6.17 & 45 \\
\hline D2 & 6.6 & 0.015 & 525 & Yes & $2.31(\times)$ & $4.20(+)$ & $1.07(\times)$ & $3.50(+)$ & 6.87 & 82 \\
\hline E0 & 6.8 & 0 & 510 & Yes & $3.44(\times)$ & $2.56(\times)$ & $2.03(\times)$ & $2.06(\times)$ & 6.60 & 88 \\
\hline E1 & 6.8 & 0.01 & 425 & Yes & $2.94(\times)$ & $2.33(\times)$ & $0.94(+)$ & $0.96(\times)$ & 6.67 & 150 \\
\hline $\mathrm{E} 2$ & 6.8 & 0.015 & 410 & Yes & $2.34(\times)$ & $3.49(\times)$ & $9.69(\times)$ & $1.69(+)$ & 5.44 & 168 \\
\hline
\end{tabular}

Note. $-L_{\nu_{e}}$ denotes the input luminosity. $\Delta t$ represents the simulation time. $\beta_{\phi}$ is a rotational parameter introduced in equation (9). $h_{\max }^{\text {pol }}, h_{\max }^{\text {equ }}$ represents the maximum amplitudes (neutrino + matter) during the simulation time seen from the pole or the equator, respectively, while $h_{\nu, \max }^{\text {pol }}, h_{\nu, \max }^{\text {equ }}$ are the ones including only the neutrino contribution. The polarization of the GWs at the maximum is indicated by + and $\times . E_{\mathrm{GW}}$, is the radiated energy in the form of the neutrino GWs in unit of $M_{\odot} c^{2} . \alpha_{\mathrm{sk}}$ is the spin-kick angle (see text for more detail). The distance to the source is assumed to be $10 \mathrm{kpc}$. 


\section{Results}

First of all, we summarize how the inclusion of rotation could have impacts on the hydrodynamics as well as the overall trends in the GW emission in section 3.1. Then in section 3.2 , we move on to analyze the gravitational waveforms more in detail.

\subsection{Stochastic Hydrodynamics and GWs}

Figures 1,2 show the 3D hydrodynamic features of the SASI near at the shock breakout from the outer boundary of the computational domain. Comparing the top left (model B2) to the bottom left panel (model C2) in Figure 1, it can be inferred that the direction of explosion varies models to models. Given the same initial angular momentum, the major axis of the growth of SASI happens to be toward the rotational axis for model B2 (top left panel showing the propagation of the high entropy bubbles (colored by red) closely parallel to the rotational axis (:z-axis)), while it is shown to be toward the equatorial direction $(: y$ axis) for model $\mathrm{C} 2$ (bottom left). A common feature seen for the pair models is the spiral flows inside the shock that rotates globally around the $z$-axis, which is seen like arcs (right panels). This is as a result of introducing the rotation whose axis corresponded to the $z$-axis. As a faster rotation was added to the flows, spiral flows run with higher velocity from the triple points that are formed inside the standing shock, to near the PNS, and then flows rotate with higher velocity inside the high-entropy blobs.

Figure 2 shows the hydrodynamic features for models with higher neutrino luminosities (models D0 (top) and E2 (bottom)). Due to the higher neutrino luminosity, the volumes of high entropy blobs (colored by red) behind the shock become much larger than the ones in Figure 1 (right panels). Some spiral structures are depicted in the bottom right panel of Figure 2, however, it is more difficult to see the structures compared to Figure 1. Observing from the horizontal direction, model D0 explodes in a rather spherical manner (top left), while the major axis of SASI for model E2 is tilted about 45 degrees from the rotational axis (bottom left). Our 3D results presented here imply that the direction of explosion as well as the major axis of the growth of SASI cannot be predicted a priori, reflecting the chaotic fluid motions driven by the interplay of the neutrino heating, SASI, and convection. This

feature is qualitatively same as the previous 3D results but without rotation (Iwakami et al. 2008; Nordhaus et al. 2010; Wongwathanarat et al. 2010).

As the explosion dynamics becomes chaotic, there is no systematic dependence of the maximum GW amplitudes and the radiated GW energies on the input neutrino luminosities (see Table 1). In fact, the largest emitted GW energy is obtained for model B2 with the 
intermediate neutrino luminosity (see, $E_{\mathrm{GW}}$ Table 1). Regarding the polarization $(+$ or $\times$ ) of the maximum GW amplitudes, it may be hard to decipher any characteristics in Table 1 due to its messiness. But looking very carefully, one may be able to find that the polarization of the maximum GWs from neutrinos seen from the equator $\left(h_{\nu, \max }^{\mathrm{equ}}\right)$ is always plus $(+)$.

This feature can be more clearly seen in the waveform catalogues presented from Figure 3] to 6. From Figure 3, it can be seen that $h_{\nu,+}^{\text {equ }}$ (blue line) becomes prominent among others after around $t \sim 500-600 \mathrm{~ms}$ for models with rotation (middle and bottom panels). This trend seems quite general as seen in the other luminosity models (see middle and bottom panels in Figure 4 to 6). Note again that the time when the rotational flow is adjusted to touch the PNS surface is $t=400 \mathrm{~ms}$ for models of series A (and $t=100 \mathrm{~ms}$ for the other), which accounts for the time difference in the rise of $h_{\nu,+}^{\text {equ }}$ (compare Figure 3 to Figure 4 to 7 ). In the absence of rotation, the waveforms do not exhibit such a feature and vary much more stochastically (e.g., top panels in Figure 3 to 7). Comparing the bottom panels in Figures 3 to 7 , it can be seen that the increasing trend becomes more fainter as the input luminosity becomes larger. This is mainly because vigorous matter motions irradiated by intense neutrino heating smear out the growth of the spiral SASI modes as well as the structures of the spiral flows. To extract purely the rotational effects on the GWs, it is most convenient to analyze models of series A that fail to produce explosions. In the following, we take models of series A as a reference and look more in detail the reason why the stochastic nature of the GWs is broken (or weakened) due to the inclusion of rotation. 

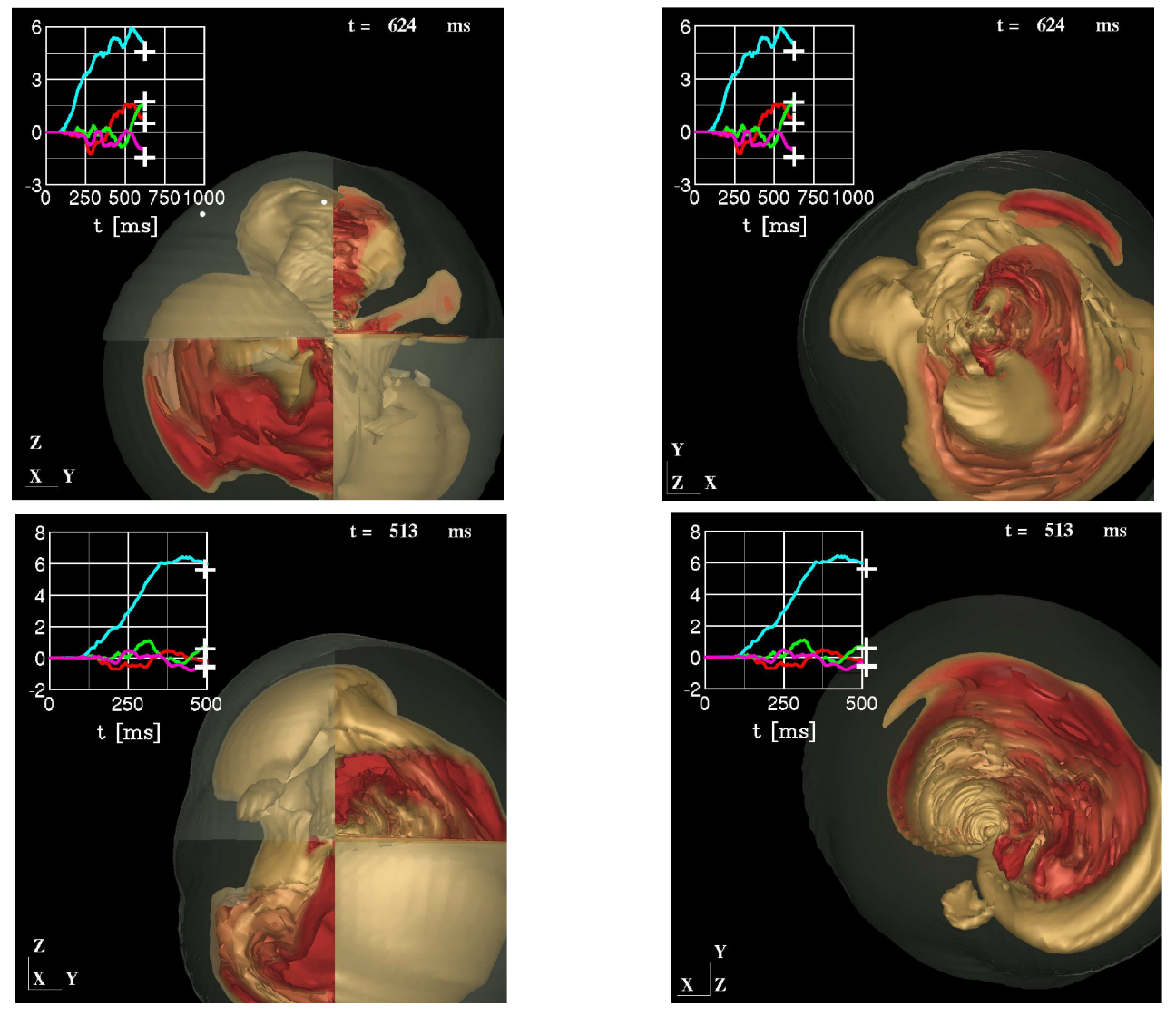

Fig. 1.- Snapshots of the entropy distributions for models B2 (top panels, $t=624 \mathrm{~ms}$ ) and $\mathrm{C} 2$ (bottom panels, $t=513 \mathrm{~ms}$ ) seen from the equator (left panels, $\phi=0$ ) or the pole (right panels, $\theta=0$ ), respectively. The second and fourth quadrant of each panel shows the surface of the standing shock wave. In the first and third quadrant, the profiles of the high entropy bubbles (colored by red) inside the section cut by the $Z Y$ (left) or $Y X$ (right) plane are shown. The side length of each plot is $1000 \mathrm{~km}$. The insets show the gravitational waveforms with ' + ' on each curves representing the time of the snapshot. Note that the colors of the curves are taken to be the same as the top panel of Figure 3 . 

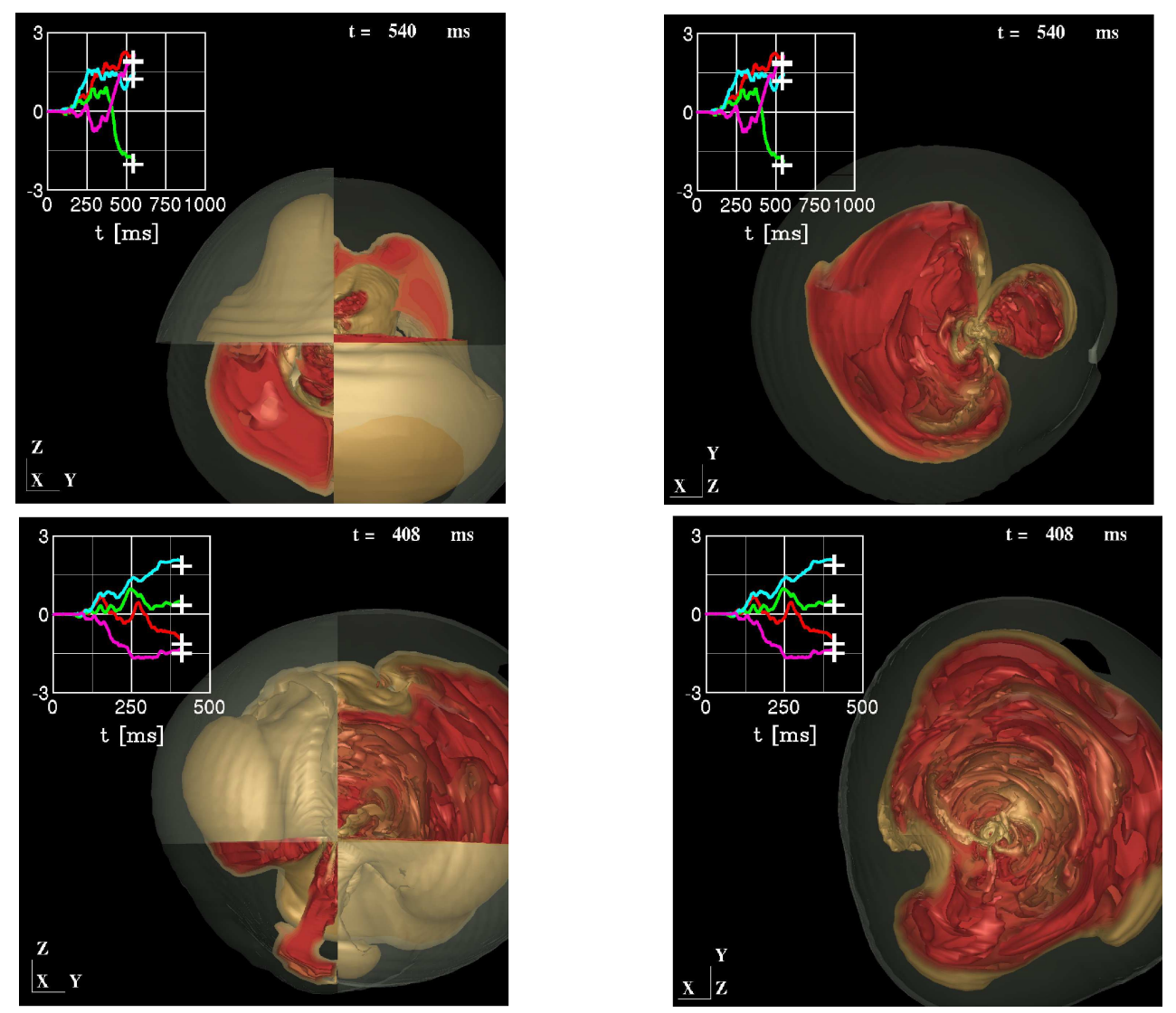

Fig. 2.- Same as Figure 1 but for models D0 (top, $t=540 \mathrm{~ms}$ ) and E2 (bottom, $t=408$ $\mathrm{ms})$. 

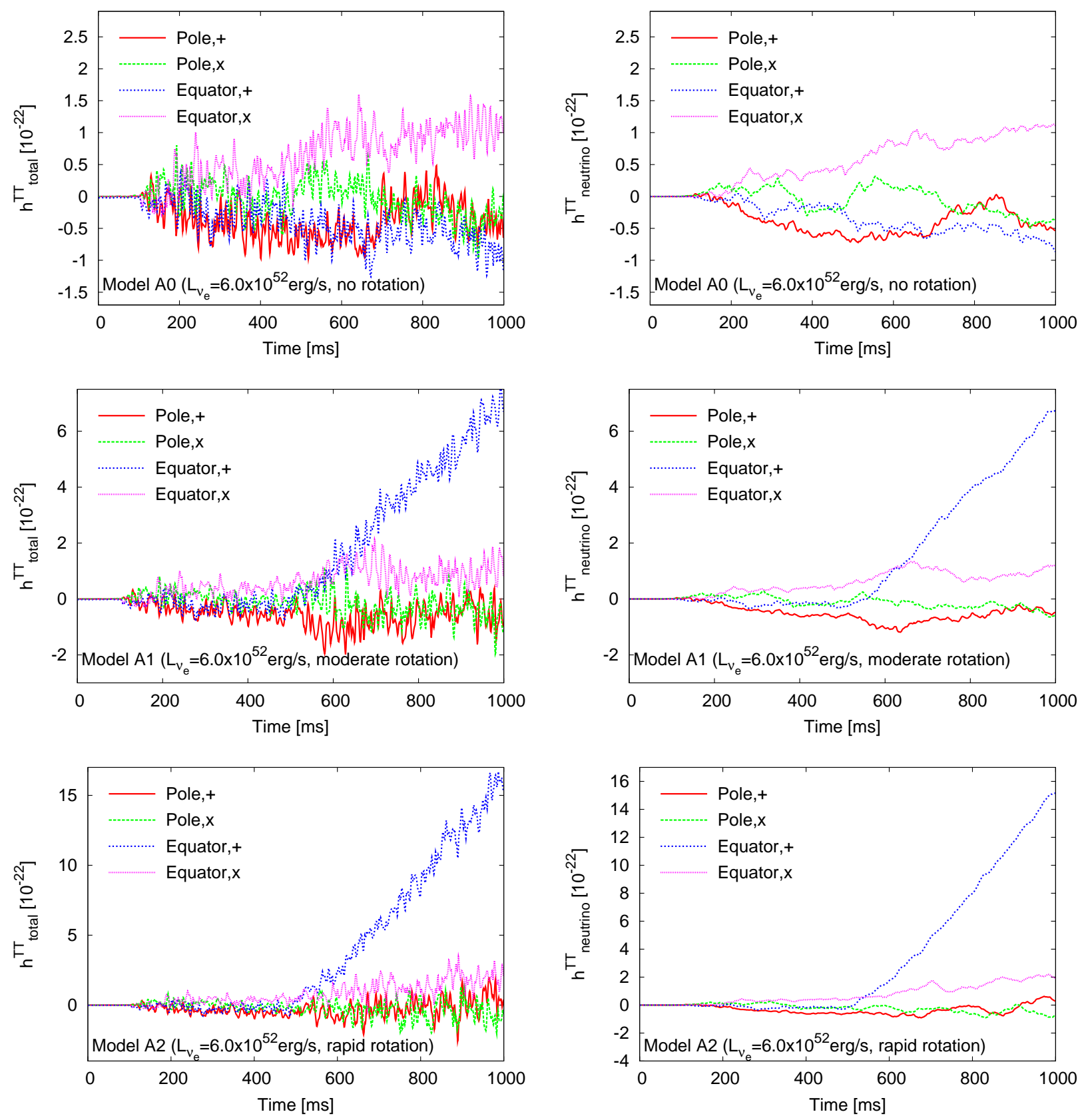

Fig. 3.- Gravitational waveforms from the sum of neutrinos and matter motions (left) and only from neutrinos (right) for models A0 (top), A1 (middle), and A2 (bottom). The time is measured from the epoch when the neutrino luminosity is injected from the surface of the neutrino sphere. In all the computed models, SASI gradually transits from the linear to non-linear regime at about $100 \mathrm{~ms}$, simultaneously making the amplitudes deviate from zero. For models of series A shown here, the rotational flow is adjusted to advect to the PNS surface at around $t=400 \mathrm{~ms}$ (see text for more detail). The supernova is assumed to be located at the distance of $10 \mathrm{kpc}$. 

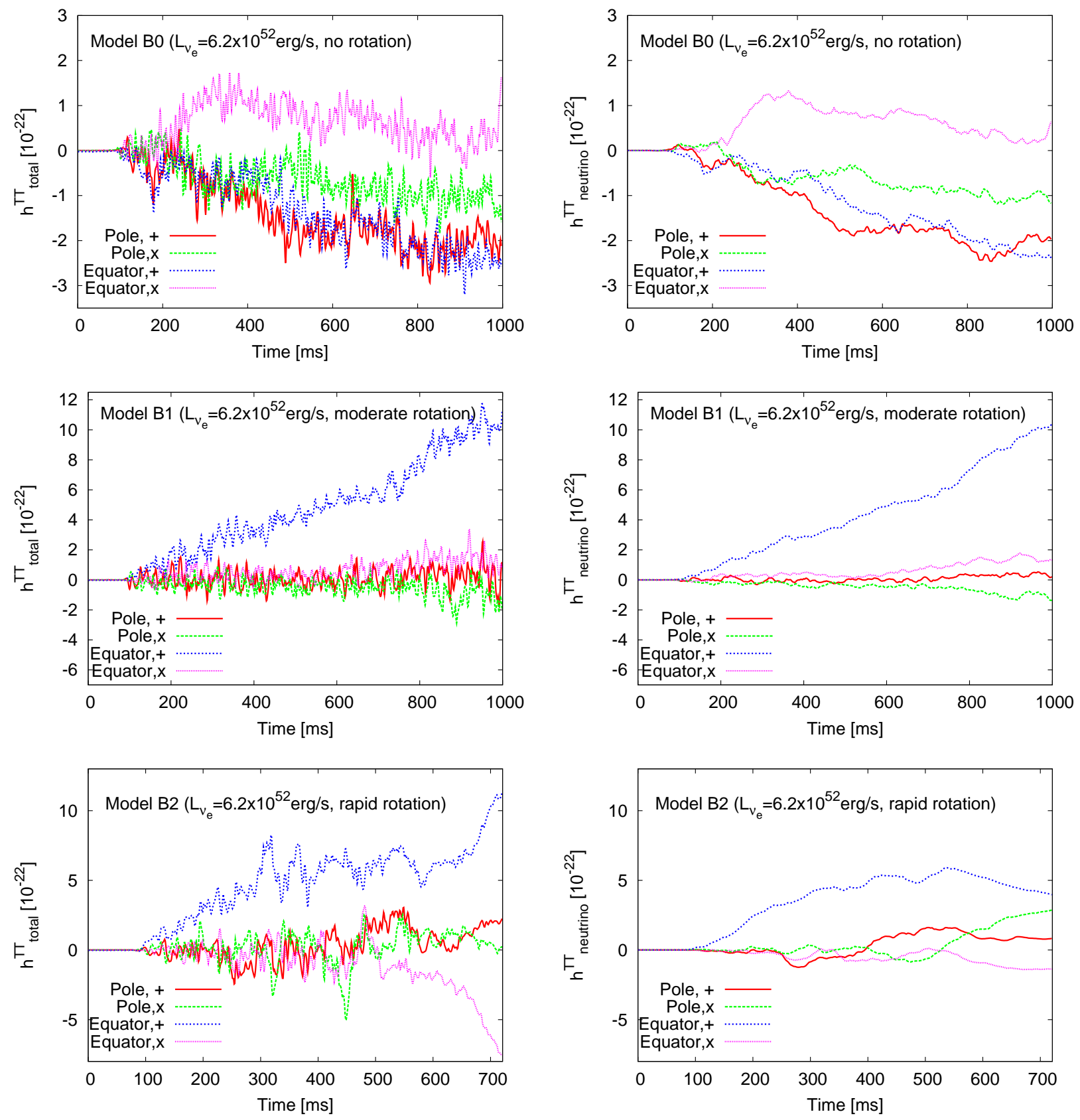

Fig. 4. - Same as Figure 3 but for models of series B . Note that except for models of series A, the rotational flows are adjusted to advect to the PNS surface at around $t=100 \mathrm{~ms}$. 

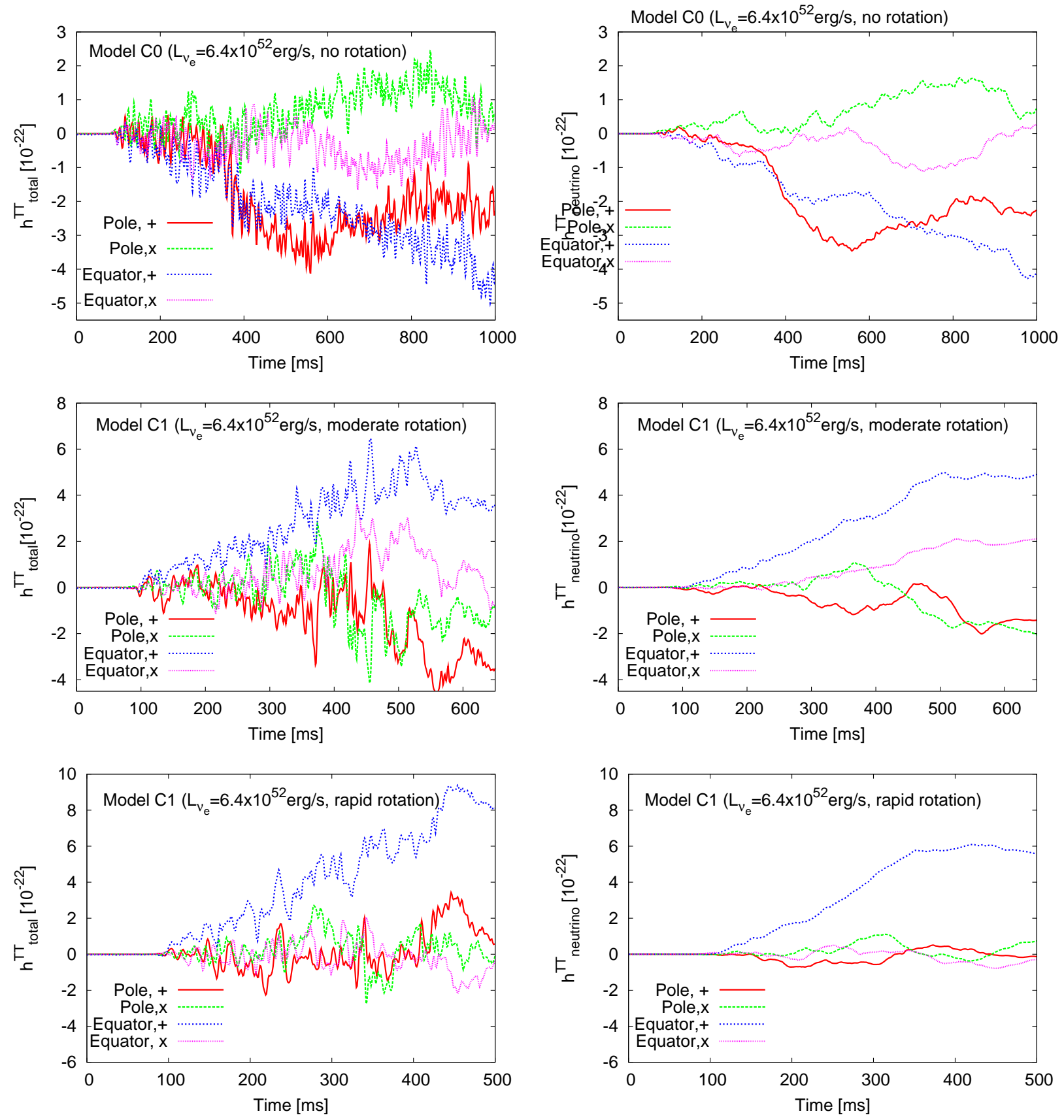

Fig. 5.- Same as Figure 4 but for models of series C. 

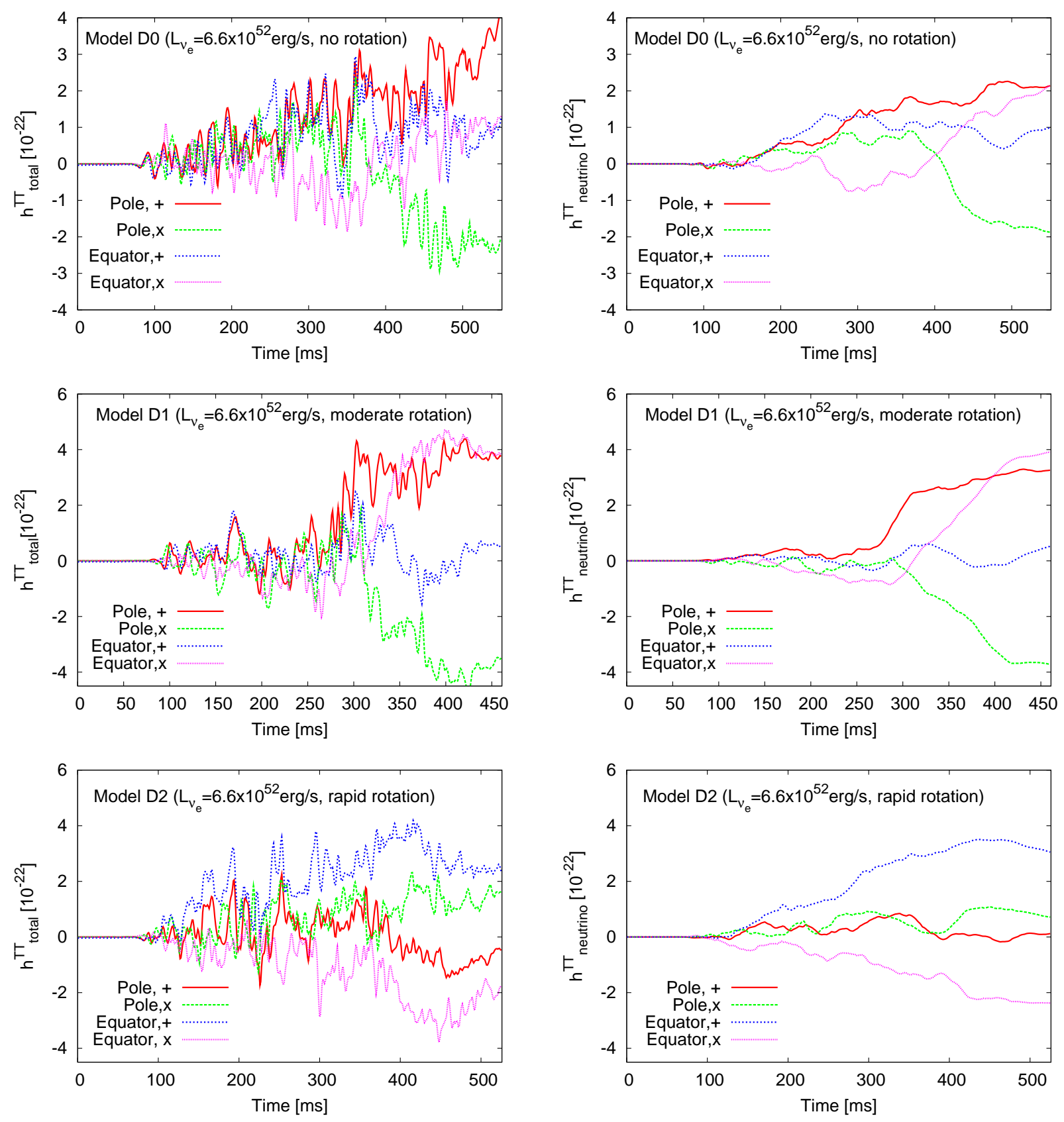

Fig. 6. - Same as Figure 4 but for models of series D. 

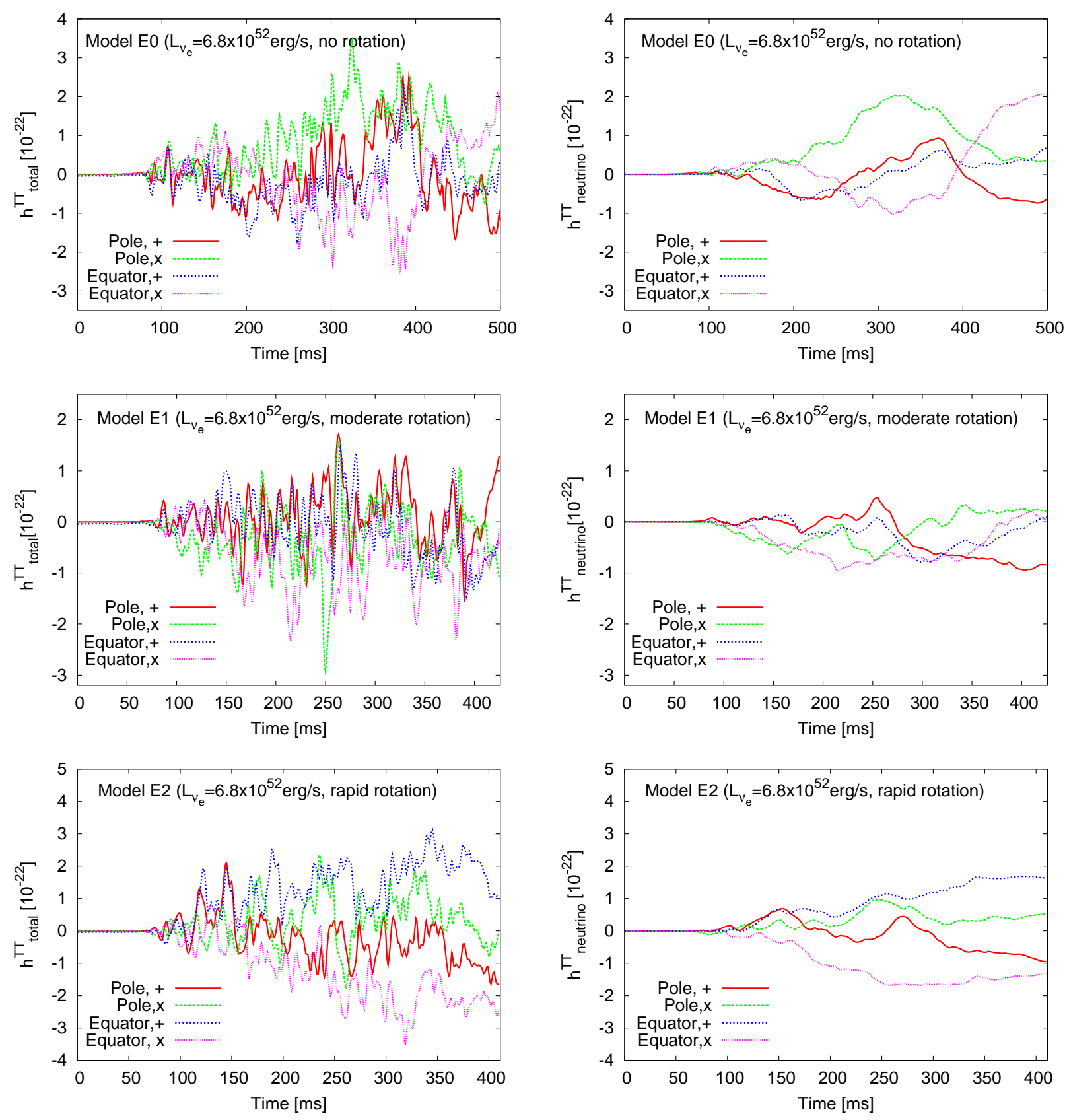

Fig. 7.- Same as Figure 4 but for models of series E. 


\subsection{Breaking of Stochasticity Due to Rotation}

Figure 8 illustrates two typical snapshots of the flow fields for model A2 before and after the rotational flow approaches to the PNS surface (left and right panels), seen from the pole (top) or from the equator (bottom), respectively. The left panels indicate that the deformation of the shock surface is only mild for this model, although the SASI has already entered the non-linear phase at around $t=100 \mathrm{~ms}$. From the bottom right panel, one may guess the presence of the sloshing modes that happen to develop along the rotational axis

( $z$-axis) at this epoch. It should be emphasized that the dominance of $h_{\nu,+}^{\text {equ }}$ observed in the current 3D simulations have nothing to do with the one found in our previous 2D studies (Kotake et al. 2009a). Free from the 2D axis effects, the major axis of the SASI changes stochastically with time, and the flow patters behind the standing shock simultaneously change in every direction. As a result, the sloshing modes can make only a small contribution to the GW emission (e.g., the inset in the left panels of Figure 8). The remaining possibility is that the spiral flows clearly seen in the top right panel should be a key importance to understand the GW feature that we pointed out in the last section.

The left panel of Figure 9 shows the local neutrino energy fluxes $\left(: d l_{\nu} /(d \Omega d S)\right.$, equation (7)) seen from the northern hemisphere. Seen as bright arcs in the left panel, the higher values of the neutrino energy fluxes are shown to coincide with the high temperature regions in the right panel. This is because the compression of matter is more enhanced in the vicinity of the equatorial plane due to the presence of the spiral flows (see velocity vectors in the right panel).

Figure 10 shows the time evolution of $d l_{\nu} / d \Omega$ (equation (8) ) in the vicinity of the north pole $(\theta=0)$, the equator $(\theta=\pi / 2)$, and the south pole $(\theta=\pi)$ (left panels), and their differences from the equator (right panels) for models A2 (top panels) and A0 (bottom panels), respectively. A common feature seen from the right panels is that the dominance of the neutrino emission in the north (red line) and south poles (green line) is occasionally anti-correlated. This is a consequence of the low-mode nature of SASI, here of $\ell=1$. The correlation shown here as well as the resulting GWs is much weaker than the ones in our 2D study (Kotake et al. (2009a)), because the major axis of the SASI changes much more randomly.

The most important message in Figure 10 is the gradual deviation of $d l_{\nu} / d \Omega$ for model A2 seen in the top right panel. This feature becomes remarkable only after $t \sim 500-600$ ms, because it takes $\sim 100-200 \mathrm{~ms}$ for the spiral SASI modes to develop behind the standing shock after the spiral flows approached to the PNS surface $(: t=400 \mathrm{~ms}$ denoted by the vertical line). As already mentioned, the dominance of the neutrino luminosity seen from the polar directions is due to the spiral flows that develop near in the vicinity of 
the equatorial plane. Comparing the two panels in Figure 11, a contrast of the neutrino luminosity in the polar regions $\left(\theta \sim 0^{\circ}\right.$ or $\left.\theta \sim 180^{\circ}\right)$ to other regions becomes prominent only after $t \sim 600 \mathrm{~ms}$ for model A2 (right panel), which is visualized as stripes bridging between the north and south pole. Without rotation, the anisotropy of neutrino radiation remains to be much smaller (left panel in Figure11), leading to much smaller GW amplitudes. Remembering again that $\Phi\left(\theta^{\prime}\right)$ in equation (3) is positive near the north and south polar caps, the dominance of the polar neutrino luminosities leads to make the positively growing feature in $h_{\nu,+}^{\text {equ }}$, so far depicted in models that include rotation (e.g., Figures 3-77). It is noted here that the lateral-angle $(\theta)$ dependence of equation (1) can be approximated by equation (3) because the neutrino anisotropy in the azimuthal direction is much weaker than the one in the lateral direction.

Figure 12 shows how the above GW features could or could not change if we change the strength of the initial velocity perturbation (top panels), the epoch when the initial rotational flow advects to the center (bottom left panel), and the numerical resolution (bottom right panel), respectively. In the top two panels, larger initial (velocity-) perturbations (5\%) are imposed (for model A1 (left) and A2 (right) as indicated by pert5\%) in contrast to the fiducial value of $1 \%$ (e.g., section 2.2). Due to the large perturbation, the timescale when the non-linear phase sets in, becomes typically 30 - $40 \mathrm{~ms}$ earlier compared to the fiducial models (compare the middle and bottom panels of Figure 3 to the top two panels of Figure 12). Regardless of the difference, the increasing trend of the GWs (blue lines) is shown to be unaffected. This is also the case when the rotational flow is adjusted to advect to the PNS in the linear SASI phase (bottom left panel), and when a finer numerical resolution is taken (bottom right panel). In the bottom right panel, the numerical resolution for the azimuthal direction is doubled compared to the fiducial value of 60 mesh points (indicated by "high-res" for model A2). For the bottom left panel, the initial perturbation is taken to be as small as $0.1 \%$ to make the linear phase longer. For this model, the rotational flow advects to the PNS about $t \sim 100 \mathrm{~ms}$ in the linear SASI phase, and the transition to the non-linear phase takes place at $t \gtrsim 200 \mathrm{~ms}$. As is shown, the increasing trend appears also from the linear SASI phase $(t \sim 100 \mathrm{~ms})$.

Finally Figure 13 depicts the GW spectra for model A0 (left panel) and A2 (right panel). The neutrino GWs seen from the equator (green line, right panel) for model A2 is larger than the one seen from the pole, and it is slightly larger compared to model A0 in the lower frequencies below $\sim 10 \mathrm{~Hz}$. It is true that the GW signals from neutrinos are very difficult to detect for ground-based detectors whose sensitivity is limited mainly by the seismic noises at such lower frequencies (Ando \& the TAMA Collaboration 2005; Abbott et al. 2005; Weinstein 2002; Kuroda \& LCGT Collaboration 2010). However these signals may be detectable by the recently proposed future space interferometers like Fabry-Perot type DE- 
CIGO (Kawamura et al. (2006), black line in Figure13). The sensitivity curve is taken from Kudoh et al. (2006). Contributed by the neutrino GWs in the lower frequency domains, the total GW spectrum tends to become rather flat over a broad frequency range below $\sim 100$ Hz. These GW features obtained in the context of the SASI-aided neutrino-driven mechanism are different from the ones expected in the other candidate supernova mechanism, such as the MHD mechanism (e.g., Obergaulinger et al. (2006); Takiwaki \& Kotake (2010)) and the acoustic mechanism (Ott et al. 2006a). Therefore the detection of such signals is expected to provide an important probe into the long-veiled explosion mechanism.

Regarding the GW amplitudes from matter motions, we find no significant change or increase due to the spiral flows either seen from the polar or equatorial directions. This is mainly because the spiral flows can develop only outside the PNS taken to be $50 \mathrm{~km}$ in our idealized simulations, leading to have small changes in the mass-quadrupole (due to its small masses). This situation might be akin to the one observed in the early $3 \mathrm{D}$ simulations by Rampp et al. (1998). To produce sizable GW amplitudes, the non-axisymmetric instabilities that globally develop in the more central regions to the PNS should be needed (e.g., Ott et al. (2007b); Scheidegger et al. (2010)). To explore these fascinating phenomena, one apparently needs to perform full 3D radiation-hydrodynamic simulations covering the entire stellar core and starting from gravitational collapse to explosions in a consistent manner. 

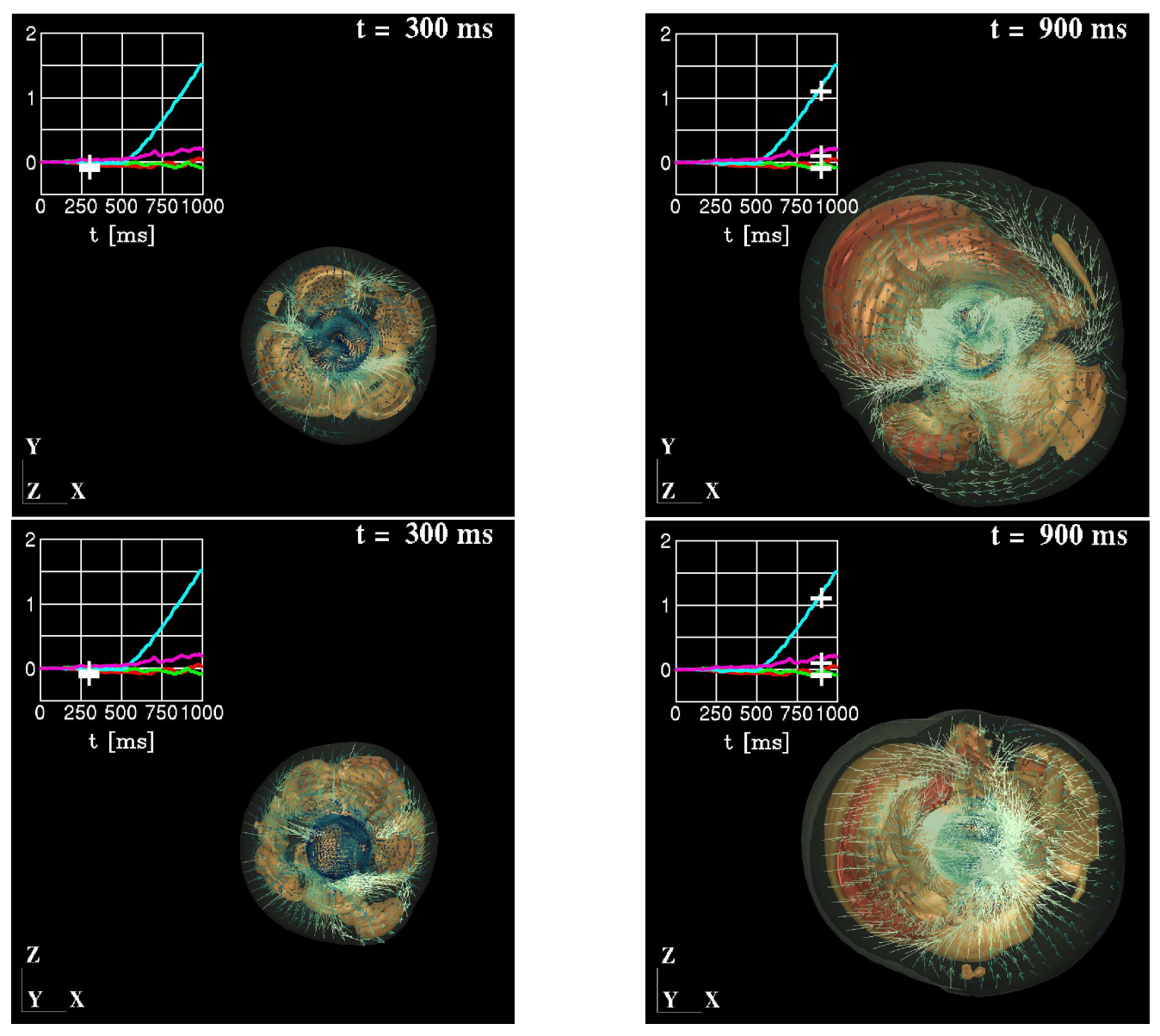

Fig. 8.- Partial cutaway of the entropy isosurfaces and the velocity vectors on the cutting plane for model A2. Left and right panels are at $t=300 \mathrm{~ms}$ and $t=900 \mathrm{~ms}$ corresponding to the epoch before and after the rotational flow approaches to the PNS surface, respectively. Top and bottom panels are for the polar and equatorial observer, respectively. The insets show the gravitational waveforms with ' + ' on each curves representing the time of the snapshot. Note that the colors of the curves are taken to be the same as the top panel of Figure 3 . 

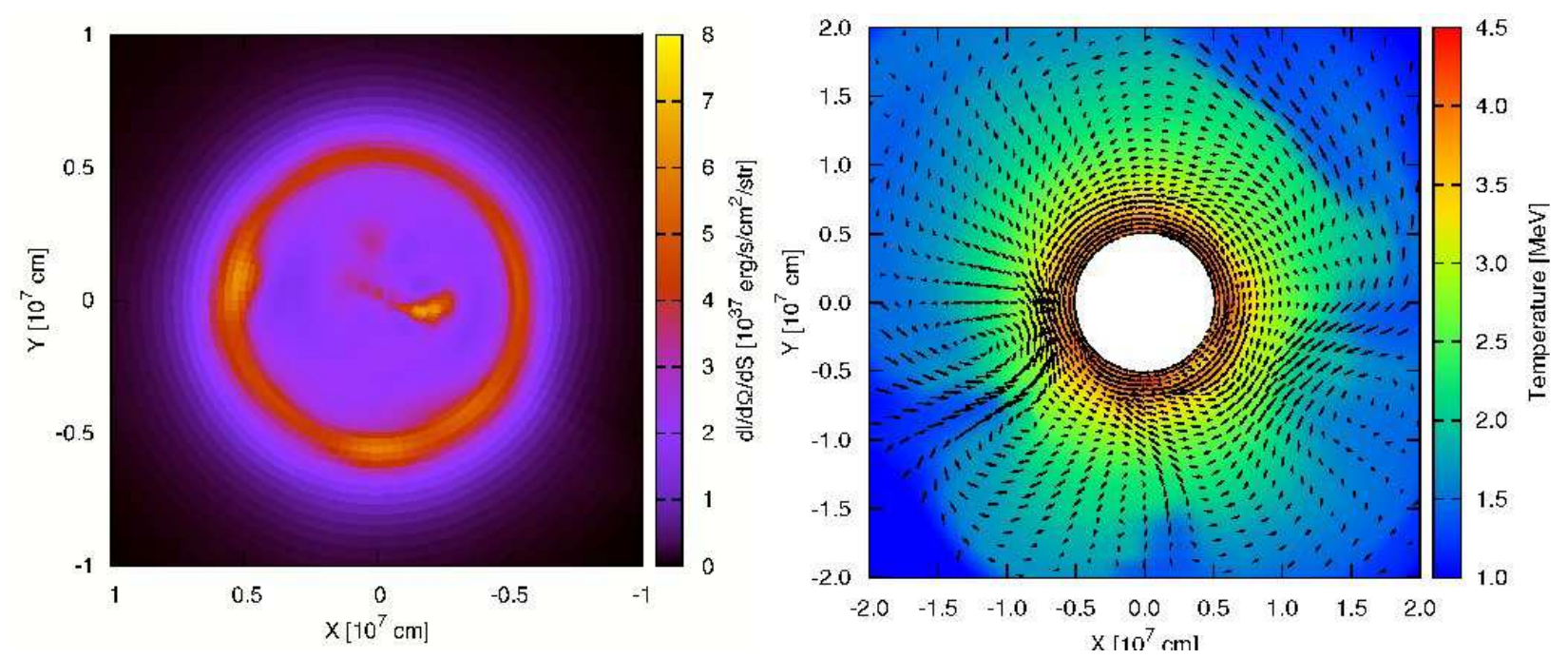

Fig. 9.- Left and right panels show the neutrino energy fluxes of $d l_{\nu} /(d \Omega d S)$ (equation(77) seen from the northern hemisphere and the temperature distributions in the equatorial plane for model A2 at $t=900 \mathrm{~ms}$.
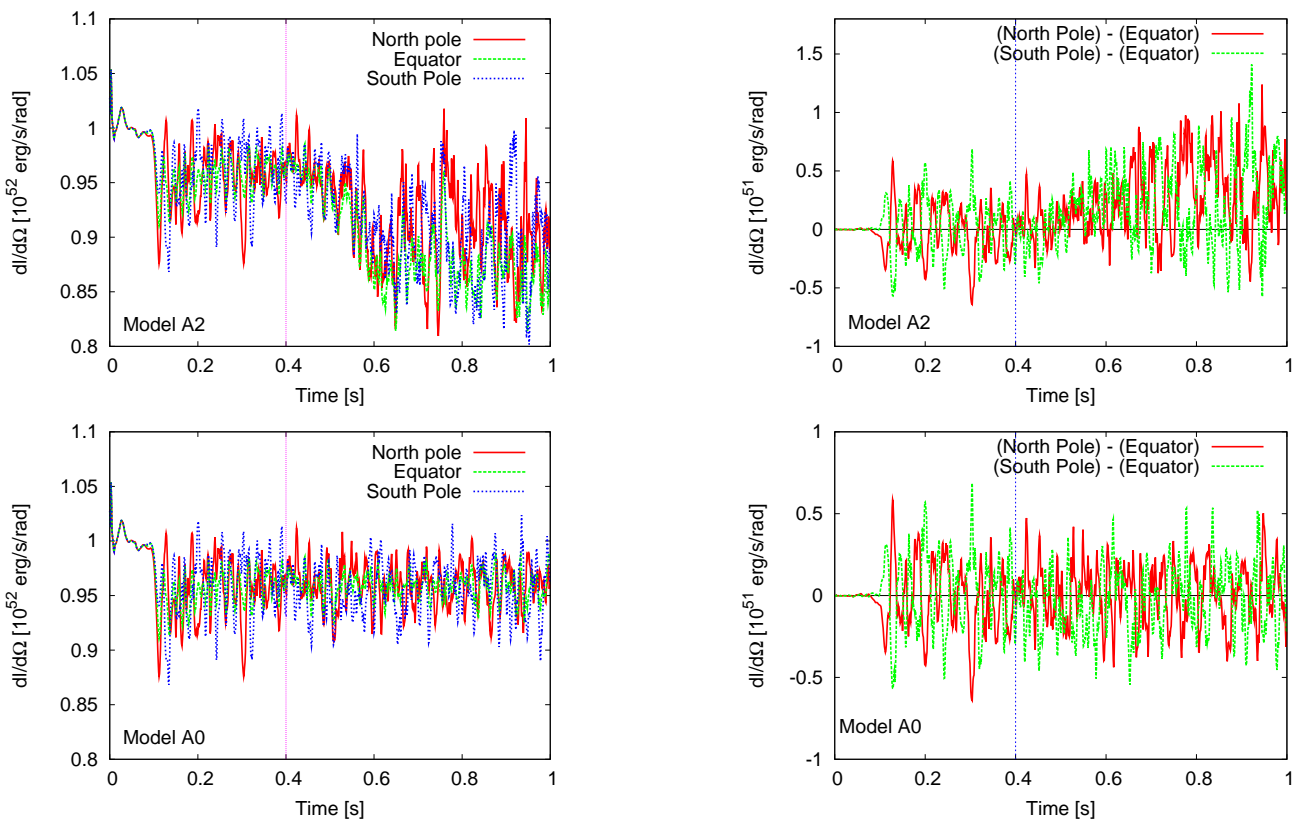

Fig. 10. - Time evolution of the directional dependent neutrino luminosity: $d l_{\nu} / d \Omega$ (left panels) in the vicinity of the north pole, the equator, and the south pole, and their differences from the equator (right panels) for models A2 (top panels) and A0 (bottom panels). Vertical lines in the right panels represent the epoch of $t=400 \mathrm{~ms}$ when the rotational flow advects to the PNS surface. 

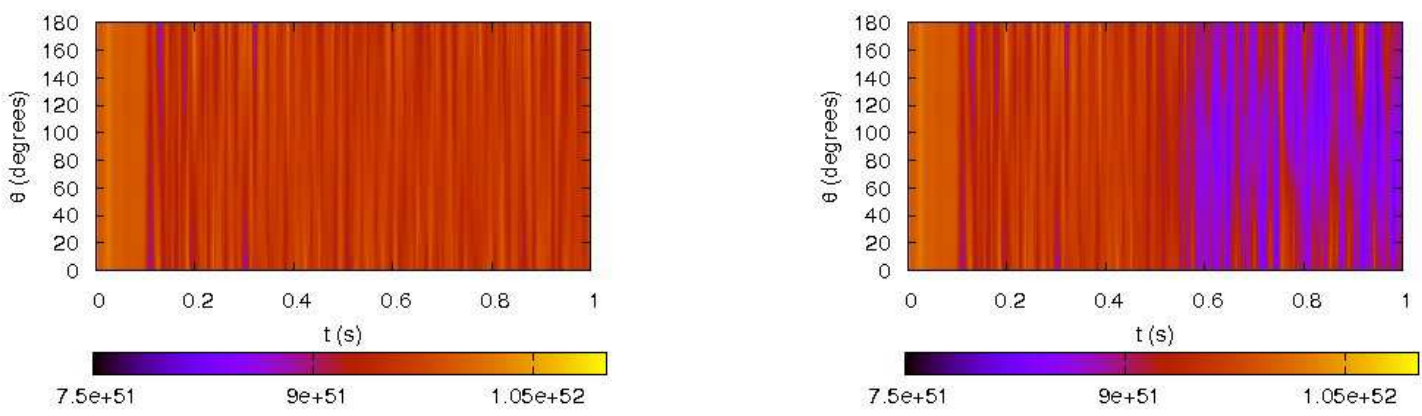

Fig. 11. - Contour of $d l_{\nu} / d \Omega$ ((azimuthal)angle-averaged) as a function of time (horizontal axis) and the polar angle (vertical axis) for models A0 (left panel) and A2 (right panel). 

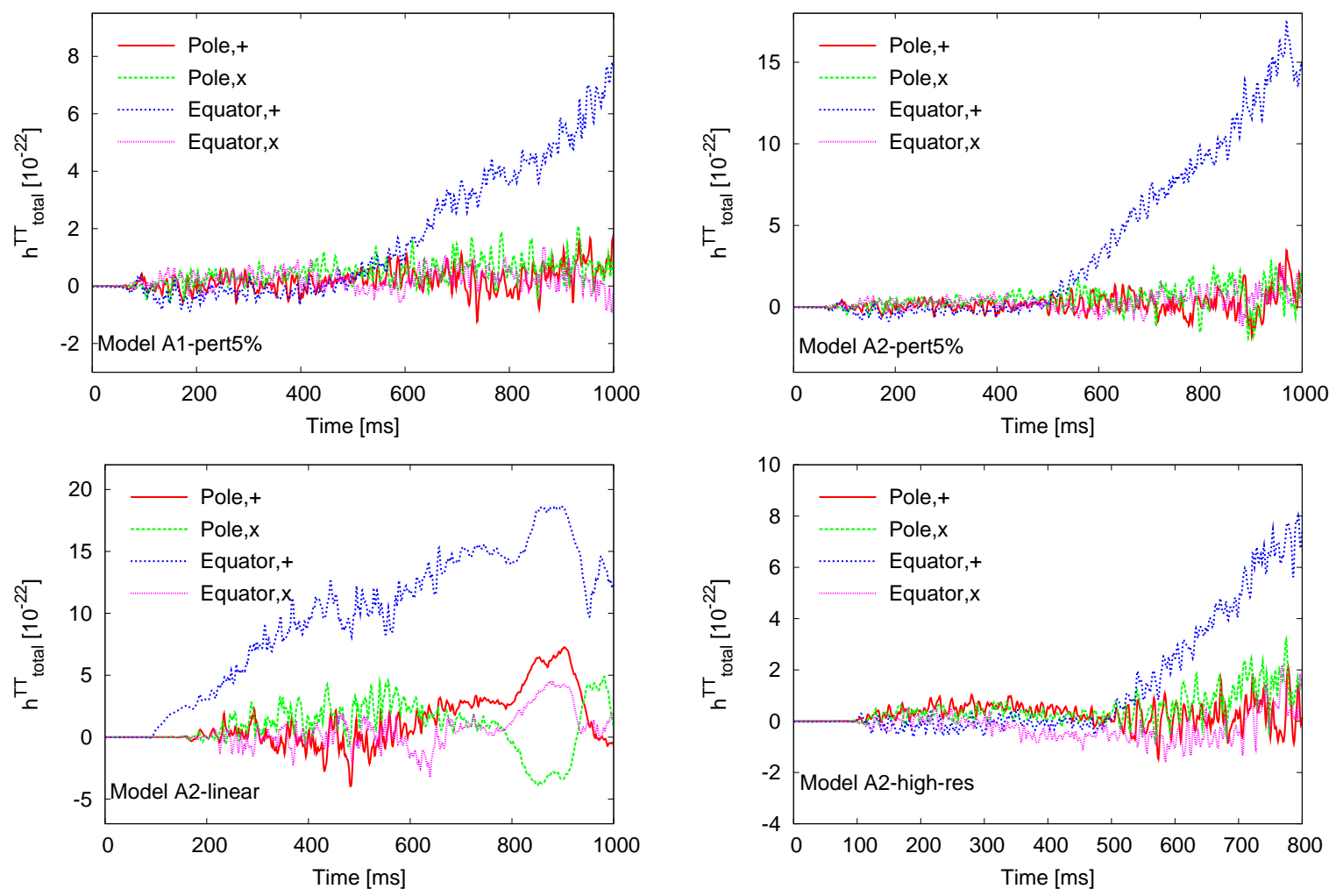

Fig. 12.- Variations of model series of A1 and A2. For top panels, larger initial (velocity-) perturbations (5\%) are imposed (for model A1 (left) and A2 (right) indicated by pert5\%) in contrast to the fiducial value of $1 \%$ (e.g., section 2.2). For the bottom left panel, the initial rotational flow is tuned to advect to the PNS surface in the linear SASI phase for model A2 (indicated by A2-linear). In the bottom right panel, the numerical resolution for the azimuthal direction is doubled compared to the fiducial value of 60 mesh points (indicated by "high-res" for model A2). 

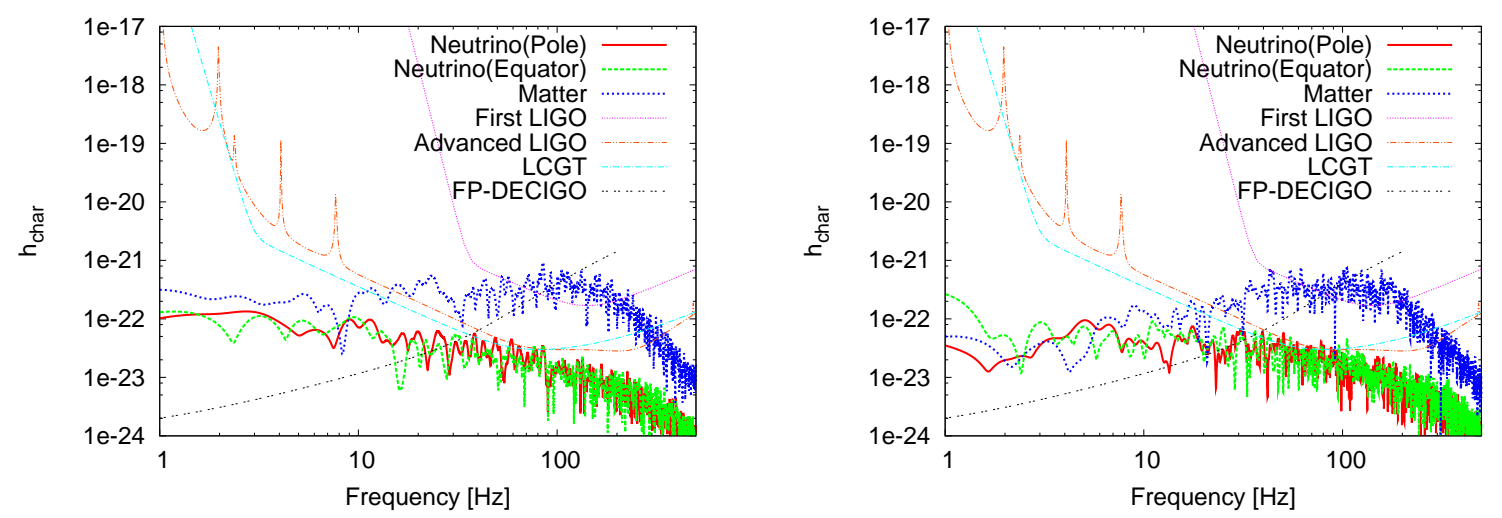

Fig. 13. - Spectral distributions of GWs from matter motions ("Matter") and neutrino emission ("Neutrino") seen from the pole or the equator for models A0 (left panel) and A2 (right panel) with the expected detection limits of TAMA (Ando \& the TAMA Collaboration 2005), first LIGO (Abbott et al. 2005), advanced LIGO (Weinstein 2002), Large-scale Cryogenic Gravitational wave Telescope (LCGT) (Kuroda \& LCGT Collaboration 2010) and Fabry-Perot type DECIGO (Kawamura et al. 2006; Kudoh et al. 2006). The distance to the supernova is assumed to be $10 \mathrm{kpc}$. Note that for the matter signal, the + mode seen from the polar direction is plotted. 


\section{Summary and Discussion}

We studied how the spiral modes of the SASI can have impacts on the properties of GWs by performing $3 \mathrm{D}$ simulations that mimic the SASI-aided core-collapse supernovae. To see the effects of rotation, we imposed a uniform rotation on the flow advecting from the outer boundary of the iron core (as in Iwakami et al. (2009)), whose specific angular momentum is assumed to agree with recent stellar evolution models. We computed fifteen 3D models in which the initial angular momentum as well as the input neutrino luminosities from the PNS are changed in a systematic manner. By performing a ray-tracing analysis, we accurately estimated the GW amplitudes from anisotropic neutrino emission. With these computations, we found that the gravitational waveforms from neutrinos in models that include rotation exhibit a common feature otherwise they vary much more stochastically in the absence of rotation. The breaking of the stochasticity stems from the excess of the neutrino emission parallel to the spin axis. This is because the compression of matter is more enhanced in the vicinity of the equatorial plane due to the growth of the spiral SASI modes, leading to the formation of the spiral flows with higher temperatures circulating around the spin axis. We pointed out that a recently proposed future space interferometers like Fabry-Perot type DECIGO would permit the detection of these signals for a Galactic supernova.

It should be noted that the approximations taken in the simulation, such as the excision inside the PNS with its fixed inner boundary and the light bulb approach with the isentropic luminosity constant with time, are only a very first step towards realistic 3D supernova simulations. As already mentioned, the excision of the central regions inside PNSs hinders the efficient gravitational emission there, such as by the g-mode oscillations (Ott et al. 2006a) and the non-axisymmetric instabilities (Ott et al. 2007b; Scheidegger et al. 2010) of the PNSs, and the enhanced neutrino emissions inside the PNSs (Marek et al. 2009). It should also affect the peak frequency of the GW spectra as pointed out by Murphy et al. (2009). The rotational flows advecting into the inner core should spin up the PNS, which is expected to conversely affect the dynamics outside (Rantsiou et al. 2010). These important feedback should be taken into account to unravel the effect of rotation more precisely. Remembering these caveats in mind, one encouraging news to us is that the gravitational waveforms obtained in the 2D radiation-hydrodynamic simulations (Yakunin et al. 2010) are similar to the ones obtained in our 2D study (Kotake et al. 2009a). It is also noted that magnetic effects should have impacts not only on the growth of the SASI (Endeve et al. 2010; Guilet et al. 2010), but also on the GW waveforms, which are remained to be studied in the 3D MHD simulations. Nowadays, the next generation 3D results are being reported by using

new simulation techniques such as the Yin-Yang grids (Wongwathanarat et al. 2010) and the adaptive mesh refinement approach (Nordhaus et al. 2010). We hope that our results may give a momentum to theorists for making the GW prediction with a better quantitative 
precision based on the sophisticated 3D supernova modeling.

K.K. would like express thanks to K. Sato and S. Yamada for continuing encouragements. Numerical computations were in part carried on XT4 and general common use computer system at the center for Computational Astrophysics, CfCA, the National Astronomical Observatory of Japan. This study was supported in part by the Grants-in-Aid for the Scientific Research from the Ministry of Education, Science and Culture of Japan (Nos. 19540309 and 20740150). 


\section{REFERENCES}

Abbott et al., M. 2005, Phys. Rev. D, 72, 122004

Andersson, N., Ferrari, V., Jones, D. I., Kokkotas, K. D., Krishnan, B., Read, J. S., Rezzolla, L., \& Zink, B. 2010, General Relativity and Gravitation, 156

Ando, M. \& the TAMA Collaboration. 2005, Classical and Quantum Gravity, 22, 881

Blondin, J. M. \& Mezzacappa, A. 2007, Nature, 445, 58

Blondin, J. M., Mezzacappa, A., \& DeMarino, C. 2003, Astrophys. J., 584, 971

Bruenn, S. W. 1985, ApJS, 58, 771

Bruenn, S. W., Mezzacappa, A., Hix, W. R., Blondin, J. M., Marronetti, P., Messer, O. E. B., Dirk, C. J., \& Yoshida, S. 2010, ArXiv e-prints

Burrows, A., Dessart, L., Livne, E., Ott, C. D., \& Murphy, J. 2007, Astrophys. J., 664, 416

Burrows, A. \& Hayes, J. 1996, Physical Review Letters, 76, 352

Burrows, A., Livne, E., Dessart, L., Ott, C. D., \& Murphy, J. 2006, Astrophys. J., 640, 878

Dimmelmeier, H., Font, J. A., \& Müller, E. 2002, Astron. Astrophys., 393, 523

Dimmelmeier, H., Ott, C. D., Janka, H.-T., Marek, A., \& Müller, E. 2007, Physical Review Letters, 98, 251101

Dimmelmeier, H., Ott, C. D., Marek, A., \& Janka, H. 2008, Phys. Rev. D, 78, 064056

Endeve, E., Cardall, C. Y., Budiardja, R. D., \& Mezzacappa, A. 2010, ApJ, 713, 1219

Epstein, R. 1978, ApJ, 223, 1037

Flanagan, É. É. \& Hughes, S. A. 1998, Phys. Rev. D, 57, 4566

Foglizzo, T., Galletti, P., Scheck, L., \& Janka, H. 2007, ApJ, 654, 1006

Fryer, C. L. 2004a, Astrophys. J. Lett., 601, L175

—. 2004b, Astrophys. J. Lett., 601, L175

Guilet, J., Foglizzo, T., \& Fromang, S. 2010, ArXiv e-prints 
Hayes, J. C., Norman, M. L., Fiedler, R. A., Bordner, J. O., Li, P. S., Clark, S. E., ud-Doula, A., \& Mac Low, M. 2006, ApJS, 165, 188

Heger, A., Woosley, S. E., \& Spruit, H. C. 2005, Astrophys. J., 626, 350

Hirata, K., Kajita, T., Koshiba, M., Nakahata, M., \& Oyama, Y. 1987, Physical Review Letters, 58, 1490

Hough, J., Rowan, S., \& Sathyaprakash, B. S. 2005, Journal of Physics B Atomic Molecular Physics, 38, 497

Iwakami, W., Kotake, K., Ohnishi, N., Yamada, S., \& Sawada, K. 2008, Astrophys. J., 678, 1207

—. 2009, Astrophys. J., 700, 232

Janka, H. \& Müller, E. 1996, A\&A, 306, 167

Kawamura, S., Nakamura, T., Ando, M., Seto, N., Tsubono, K., Numata, K., Takahashi, R., Nagano, S., Ishikawa, T., Musha, M., Ueda, K., Sato, T., Hosokawa, M., Agatsuma, K., Akutsu, T., Aoyanagi, K., Arai, K., Araya, A., Asada, H., Aso, Y., Chiba, T., Ebisuzaki, T., Eriguchi, Y., Fujimoto, M., Fukushima, M., Futamase, T., Ganzu, K., Harada, T., Hashimoto, T., Hayama, K., Hikida, W., Himemoto, Y., Hirabayashi, H., Hiramatsu, T., Ichiki, K., Ikegami, T., Inoue, K. T., Ioka, K., Ishidoshiro, K., Itoh, Y., Kamagasako, S., Kanda, N., Kawashima, N., Kirihara, H., Kiuchi, K., Kobayashi, S., Kohri, K., Kojima, Y., Kokeyama, K., Kozai, Y., Kudoh, H., Kunimori, H., Kuroda, K., Maeda, K., Matsuhara, H., Mino, Y., Miyakawa, O., Miyoki, S., Mizusawa, H., Morisawa, T., Mukohyama, S., Naito, I., Nakagawa, N., Nakamura, K., Nakano, H., Nakao, K., Nishizawa, A., Niwa, Y., Nozawa, C., Ohashi, M., Ohishi, N., Ohkawa, M., Okutomi, A., Oohara, K., Sago, N., Saijo, M., Sakagami, M., Sakata, S., Sasaki, M., Sato, S., Shibata, M., Shinkai, H., Somiya, K., Sotani, H., Sugiyama, N., Tagoshi, H., Takahashi, T., Takahashi, H., Takahashi, R., Takano, T., Tanaka, T., Taniguchi, K., Taruya, A., Tashiro, H., Tokunari, M., Tsujikawa, S., Tsunesada, Y., Yamamoto, K., Yamazaki, T., Yokoyama, J., Yoo, C., Yoshida, S., \& Yoshino, T. 2006, Classical and Quantum Gravity, 23, 125

Kotake, K., Iwakami, W., Ohnishi, N., \& Yamada, S. 2009a, Astrophys. J., 704, 951

-. 2009b, Astrophys. J. Lett., 697, L133

Kotake, K., Ohnishi, N., \& Yamada, S. 2007, Astrophys. J., 655, 406 
Kotake, K., Sato, K., \& Takahashi, K. 2006, Reports of Progress in Physics, 69, 971

Kotake, K., Yamada, S., \& Sato, K. 2003, Phys. Rev. D, 68, 044023

Kotake, K., Yamada, S., Sato, K., Sumiyoshi, K., Ono, H., \& Suzuki, H. 2004, Phys. Rev. D, 69,124004

Kudoh, H., Taruya, A., Hiramatsu, T., \& Himemoto, Y. 2006, Phys. Rev. D, 73, 064006

Kuroda, K. \& LCGT Collaboration. 2010, Classical and Quantum Gravity, 27, 084004

Marek, A. \& Janka, H.-T. 2009, Astrophys. J., 694, 664

Marek, A., Janka, H.-T., \& Müller, E. 2009, Astron. Astrophys., 496, 475

Mönchmeyer, R., Schaefer, G., Mueller, E., \& Kates, R. E. 1991, Astron. Astrophys., 246, 417

Müler, E. \& Janka, H.-T. 1997, Astron. Astrophys., 317, 140

Müller, E., Rampp, M., Buras, R., Janka, H.-T., \& Shoemaker, D. H. 2004, Astrophys. J., 603,221

Murphy, J. W., Ott, C. D., \& Burrows, A. 2009, ArXiv e-prints

Nordhaus, J., Burrows, A., Almgren, A., \& Bell, J. 2010, ApJ, 720, 694

Obergaulinger, M., Aloy, M. A., \& Müller, E. 2006, Astron. Astrophys., 450, 1107

Ohnishi, N., Kotake, K., \& Yamada, S. 2006, Astrophys. J., 641, 1018

Ott, C. D. 2009, Classical and Quantum Gravity, 26, 063001

Ott, C. D., Burrows, A., Dessart, L., \& Livne, E. 2006a, Physical Review Letters, 96, 201102

Ott, C. D., Burrows, A., Livne, E., \& Walder, R. 2004, Astrophys. J., 600, 834

Ott, C. D., Burrows, A., Thompson, T. A., Livne, E., \& Walder, R. 2006b, Astrophys. J., Suppl. Ser., 164, 130

Ott, C. D., Dimmelmeier, H., Marek, A., Janka, H., Hawke, I., Zink, B., \& Schnetter, E. 2007a, Physical Review Letters, 98, 261101

—. 2007b, Physical Review Letters, 98, 261101 
Ott, C. D., Dimmelmeier, H., Marek, A., Janka, H., Zink, B., Hawke, I., \& Schnetter, E. 2007c, Classical and Quantum Gravity, 24, 139

Rampp, M., Mueller, E., \& Ruffert, M. 1998, A\&A, 332, 969

Rantsiou, E., Burrows, A., Nordhaus, J., \& Almgren, A. 2010, ArXiv e-prints

Scheck, L., Kifonidis, K., Janka, H., \& Müller, E. 2006, A\&A, 457, 963

Scheck, L., Plewa, T., Janka, H.-T., Kifonidis, K., \& Müller, E. 2004, Physical Review Letters, 92, 011103

Scheidegger, S., Käppeli, R., Whitehouse, S. C., Fischer, T., \& Liebendörfer, M. 2010, A\&A, $514, \mathrm{~A} 51+$

Shibata, M. \& Sekiguchi, Y.-I. 2004, Phys. Rev. D, 69, 084024

Suwa, Y., Kotake, K., Takiwaki, T., Whitehouse, S. C., Liebendoerfer, M., \& Sato, K. 2009, ArXiv e-prints

Takiwaki, T. \& Kotake, K. 2010, ArXiv e-prints

Takiwaki, T., Kotake, K., Nagataki, S., \& Sato, K. 2004, Astrophys. J., 616, 1086

Takiwaki, T., Kotake, K., \& Sato, K. 2009, Astrophys. J., 691, 1360

Weinstein, A. 2002, Classical and Quantum Gravity, 19, 1575

Wongwathanarat, A., Hammer, N. J., \& Müller, E. 2010, A\&A, 514, A48+

Yakunin, K. N., Marronetti, P., Mezzacappa, A., Bruenn, S. W., Lee, C., Chertkow, M. A., Hix, W. R., Blondin, J. M., Lentz, E. J., Bronson Messer, O. E., \& Yoshida, S. 2010, Classical and Quantum Gravity, 27, 194005

Yamasaki, T. \& Foglizzo, T. 2008, ApJ, 679, 607

Zhuge, X., Centrella, J. M., \& McMillan, S. L. W. 1994, Phys. Rev. D, 50, 6247

Zwerger, T. \& Müller, E. 1997, Astron. Astrophys., 320, 209 